\title{
The Comparison of Methods to Assess In Vitro Drug Release from Nanoparticles
}

\author{
P. A. Grabnar ${ }^{1}$, J. KerČ ${ }^{2}$, M. Homar ${ }^{2}$, J. KrISTL ${ }^{1}$ \\ ${ }^{1}$ University of Ljubljana, Faculty of Pharmacy, 1000 Ljubljana, Slovenia \\ ${ }^{2}$ Lek Pharmaceuticals d. d., Research and Development, 1000 Ljubljana, Slovenia \\ E-mail: ahlinp@ffa.uni-lj.si (P. A. Grabnar)
}

Sci Pharm. 2010; 78: 679

doi:10.3797/scipharm.cespt.8.PNM09

In studying in vitro drug release from nanoparticles (NPs) an important issue to consider is the method that can be used to measure drug release [1]. The characterization of the in vitro drug release from a colloidal carrier is technically difficult to achive. This can be attributed to the inability of effective and rapid separation of the particles from the dissolved or released drug in the surrounding medium owing to the very small size of nanocarriers [2].

The aim of our study was to compare in vitro release profiles of budesonide from NPs, which were obtained using four different methods for the assessment of drug release: membrane diffusion technique (dialysis or reverse dialysis) and sample and separate technique (filtration or ultracentrifugation).

Budesonid as a hydrophobic drug was incorporated into poly(lactic acid) NPs, which were prepared by the nanoprecipitation method using vibrating nozzle device [3]. The mean size of NPs was $260 \mathrm{~nm}$, the entrapment efficiency of budesonid $70 \%$ and drug content $5,6 \%$. Our results show that in vitro drug release profiles from NPs obtained by four methods were different. In vitro release profiles obtained by filtration, centrifugation and reverse dialysis exhibited a typical biphasic release phenomenon namely initial burst release and consequently sustained release. The critical points in filtration are the pore size and adsorption of dissolved drug on filter membrane. The problem with centrifugation is that smallest particles are very slow to sediment and further dissolution can occur over this time. The fastest burst release phase was obtained after centrifugation following by filtration and reverse dialysis. The release of budesonide from NPs determined by dialysis was much slower and showed no burst release. In this method the transport across the membrane was rate limiting, and therefore the true release rate was not measured.

No clear agreement exists on the suitability of the techniques reported to date for drug release determination from particles in the nanometer size range. Our results show that the comparison of in vitro release results obtaned by different methods is not reasonable.

[1] D'Souza S.S, DeLuca P.P. Methods to assess in vitro drug release from injectable polymeric particulate systems. Pharm Res. 2006; 23: 460-474. doi:10.1007/s11095-005-9397-8

[2] Dolenc A, Kristl J, Baumgartner S, Planinsek O. Advantages of celecoxib nanosuspension formulation and transformation into tablets. Int J Parm. 2009; 376: 204-212. doi:10.1016/j.jpharm.2009.04.038

[3] Zvonar A, Kristl J, Kerč J, Ahlin Grabnar P. High celecoxib-loaded nanoparticles prepared by a vibrating nozzle device. J Microencaps. 2009; 26: 748-759. doi:10.3109/02652040802584402 